\title{
Protein-based soft micro-optics fabricated by femtosecond laser direct writing
}

\author{
Yun-Lu Sun ${ }^{1}$, Wen-Fei Dong ${ }^{1}$, Li-Gang Niu ${ }^{1}$, Tong Jiang ${ }^{1}$, Dong-Xu Liu ${ }^{1}$, Lu Zhang ${ }^{1}$, Ying-Shuai Wang ${ }^{1}$, \\ Qi-Dai Chen ${ }^{1}$, Dong-Pyo Kim ${ }^{3}$ and Hong-Bo Sun ${ }^{1,2}$
}

In this work, we report a novel soft diffractive micro-optics, called 'microscale kinoform phase-type lens (micro-KPL)', which is fabricated by femtosecond laser direct writing (FsLDW) using bovine serum albumin (BSA) as building blocks and flexible polydimethylsiloxane (PDMS) slices as substrates. By carefully optimizing various process parameters of FsLDW (e.g., average laser power density, scanning step, exposure time on a single point and protein concentration), the as-formed protein micro-KPLs exhibit excellent surface quality, well-defined three-dimensional (3D) geometry and distinctive optical properties, even in relatively harsh operation environments (for instance, in strong acid or base). Laser shaping, imaging and other optical performances can be easily achieved. More importantly, micro-KPLs also have unique flexible and stretchable properties as well as good biocompatibility and biodegradability. Therefore, such protein hydrogel-based micro-optics may have great potential applications, such as in flexible and stretchable photonics and optics, soft integrated optical microsystems and bioimplantable devices.

Light: Science \& Applications (2014) 3, e129; doi:10.1038/Isa.2014.10; published online 17 January 2014

Keywords: femtosecond laser direct writing; micro-optics; protein; soft

\section{INTRODUCTION}

Soft micro-optics, which can be twisted, folded, compressed and stretched repeatedly without any significant damage to mechanical or optical characteristics, have become increasingly important in applications such as adaptive microlenses, ${ }^{1}$ paper-like displays, ${ }^{2,3}$ solar cells, ${ }^{4-6}$ photonic integrated systems ${ }^{7,8}$ and, most notably, bio-integrated and bio-implantable photonic micro/nanodevices. ${ }^{9-17}$ Until now, numerous fabrication methods, for instance, transferring-andprinting processes, hot-embossing technique, nano-imprinting, and self-assembly approaches, have been developed to produce flexible and stretchable micro-optics using organic, polymer, silk or hydrogel materials. ${ }^{13-20}$ Owing to their abundance, renewability, inexpensiveness, degradability, biocompatibility and unique functionality, protein-based soft micro-optics demonstrate superior performances that exceed many devices made from currently available organic materials. However, developing a convenient, economic and ecofriendly fabrication strategy that could readily produce high-performance protein-based soft micro-optics with high accuracy and improved quality is challenging. Toward this end, the novel femtosecond laser direct writing (FsLDW) method offers compelling advantages; it is a facile, rapid, non-contact and maskless approach that can realize arbitrary, designable and complicated architectures with a nanometric resolution, which conventional methods fail to provide. ${ }^{21-24}$
Recently, it has been demonstrated that biocompatible protein micro/nanoscale structures and devices (e.g., micro-optics) could be successfully fabricated by FsLDW techniques. ${ }^{25-31}$ Due to the low collateral damage of FsLDW, which achieved by high spacial restriction of the laser energy and a Fs-scale pulse width that is much shorter than the thermal diffusion time, these protein microstructures exhibit unique bioactivities, ${ }^{25}$ good biocompatibility and good tunability under appropriate external stimuli. ${ }^{26,27}$ However, the quality of protein micro-optics has been highly improved by optimized laser processing and the self-smoothing effect after two-photon-induced polymerization, which ensures their high performance optical properties. ${ }^{26,27}$ Despite the significant progress that has been achieved to date, the existing protein micro-optics have not taken full advantage of their soft features (both flexibility and stretchability) and have not been applied as soft micro-optics, which ultimately limits their further applications. For this reason, in this work, we will report a soft diffractive micro-optics, called 'microscale kinoform phase-type lenses (micro-KPL)', fabricated by FsLDW using bovine serum albumin (BSA) as building blocks and flexible polydimethylsiloxane (PDMS) slices as substrates. The protein soft micro-optics exhibit not only unique flexible and stretchable properties, but also good biocompatibility and biodegradability. Furthermore, the long-term stability of the optical profile and surface morphology of micro-KPLs can be

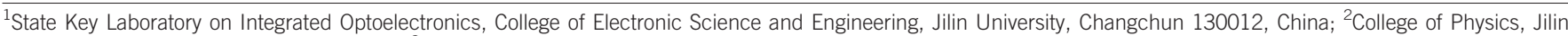

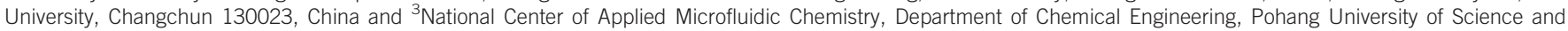
Technology, Pohang 790-784, Korea

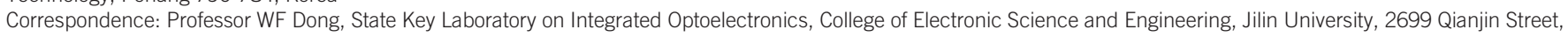
Changchun 130012, China

E-mail: wenfeidong@126.com

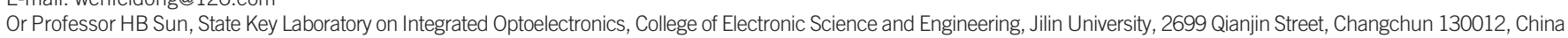
E-mail: hbsun@jlu.edu.cn

Received 1 May 2013; revised 5 September 2013; accepted 16 September 2013 
demonstrated in air, pure water and even a strong acid or base solution. All of these merits give the protein micro-KPLs great potential for practical applications as an environment-friendly soft micro-optics.

\section{MATERIALS AND METHODS}

\section{Preparation of the protein aqueous ink for FsLDW}

Appropriate amounts of BSA (product no. A7638; Sigma-Aldrich) and methylene blue (product no. M9140; Aldrich, Sigma-Aldrich, Co. 3050 Spruce Street, St. Louis. MO 63103 USA.) were dissolved in phosphate buffer $(\mathrm{pH}=7.4)$ to obtain a protein aqueous ink containing $600 \mathrm{mg} \mathrm{mL}^{-1}$ BSA and $0.6 \mathrm{mg} \mathrm{mL}^{-1}$ methylene blue. The solution needed to be incubated for several hours at $4{ }^{\circ} \mathrm{C}$ for sufficient dissolution of reagents. Ultrapure water $\left(18.2 \mathrm{M} \Omega \mathrm{cm}, 25^{\circ} \mathrm{C}\right)$, obtained using a MILLIPORE water purification system, was used in the experiment.

\section{Preparation of buffers with different $\mathrm{pH}$ values}

First, $0.71 \mathrm{~g}$ of $\mathrm{NaH}_{2} \mathrm{PO}_{4}(\mathrm{Mr}=142)$ and $2.181 \mathrm{~g}$ of $\mathrm{NaCl}$ were dissolved in approximately $450 \mathrm{~mL}$ of ultrapure water. Then, the solution was titrated to $\mathrm{pH}$ 7.41, as assessed by a $\mathrm{pH}$ meter (PB-21; Sartorius, Sartoriu AG, Weender Landstr. 94-108 37075 Goettingen, Germany), with a high-concentration $\mathrm{NaOH}$ aqueous solution. In addition, the volume was increased to $500 \mathrm{~mL}$ with ultrapure water. All the procedures were conducted at room temperature $\left(\sim 22{ }^{\circ} \mathrm{C}\right)$. Thus, in the end, the buffer had a $\mathrm{pH}$ value of 7.40 , and the ionic strength was $0.1 \mathrm{M}$ at room temperature $\left(\sim 22{ }^{\circ} \mathrm{C}\right)$. Phosphate buffers with ionic strength $0.1 \mathrm{M}$ and $\mathrm{pH}$ values from 1.0 to 13.0 were obtained by similar procedures.

\section{FsLDW of protein micro-KPLs}

KPLs are high-efficiency phase-type diffractive lenses that consist concentrically of several odd and even zones. ${ }^{32}$ In this work, protein micro-KPLs are composed of three odd and three even zones, as shown in Figure 1c and Figure 2a-1. The outer radius $r_{m}$ of the $m$ th zone (counting from the inside) of the protein micro-KPLs can be determined by the equation $r_{m}^{2}+f^{2}=(f+m \lambda / N)^{2}$, where $f$ is the designed focal length, $\lambda$ is the wavelength of light in vacuum and $N$ is the level of micro-KPLs. ${ }^{32}$ Here, $N=2^{L}$, where $L$ is the number of layers of the micro-KPL. In this work, $L=1$ and $N=2$. Because $N=2$ and $m \lambda$ is much smaller than $f$, the formula can be rewritten as $f \approx r_{m}^{2} / m \lambda$. Thus, $r_{m}$ and $\lambda$ strongly affect $f$. Because $f$ is inversely proportional to $\lambda, \mathrm{KPL}$ is a type of lens element with typical minus chromatic aberration. When light is passing through all zones, it will be interfered constructively at the focus due to the phase change induced by thickness variation. The maximum intensity is located at the focal spot. However, the diffraction efficiency of micro-KPLs is up to $40.5 \%$, which is three times higher than that of Fresnel zone plates (amplitude-type diffractive lenses). ${ }^{32}$ Because either the odd or the even zones of an Fresnel zone plate are opaque, much light is blocked. However, all the zones in the KPLs are transparent. ${ }^{32}$ Due to the minus chromatic aberration profile and higher efficiency of diffractive microlenses, KPLs are widely used in applications such as laser shaping, imaging, and compact integrated optical systems. ${ }^{32}$ Moreover, because a KPL's focal length is determined by $f \approx r_{m}^{2} / m \lambda, r_{m}$ and $\lambda$ are two main factors in determining $f$, and both parameters are stimuli-independent (for example, previous reports have shown that these parameters are not affected by $\mathrm{pH}$-independent). These properties help to achieve a stimuli-independent protein microlens, which might be very useful in certain cases where stimuliresponse is undesired (mainly bio-optical applications).

In our experiment, a homemade system for FsLDW was built to produce protein micro-KPLs. The aqueous mixture of BSA (600 $\left.\mathrm{mg} \mathrm{mL}^{-1}\right)$ and methylene blue $\left(0.6 \mathrm{mg} \mathrm{mL}^{-1}\right)$, as a photosensitizer, was freshly prepared as the FsLDW ink prior to the laser fabrication. The
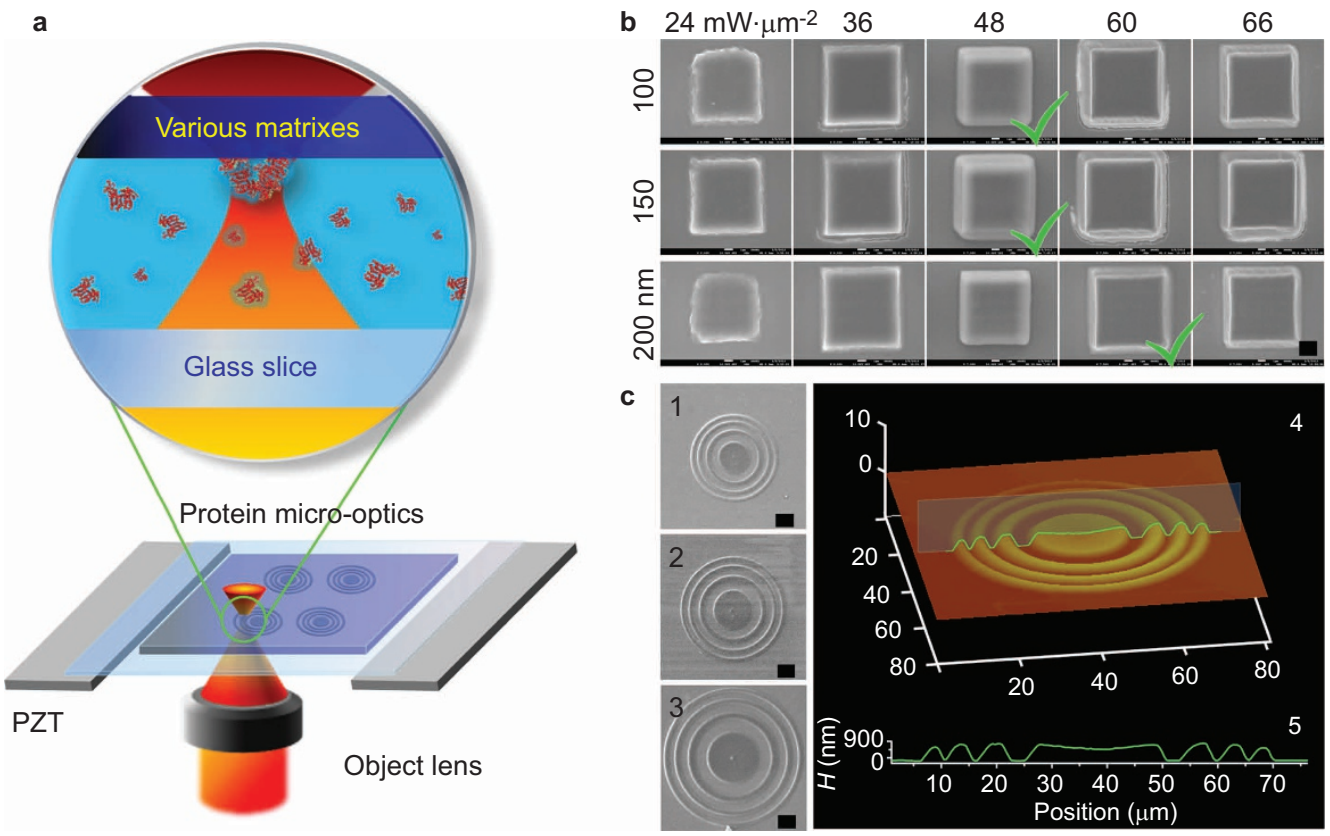

Figure 1 (a) Schematic of a homemade femtosecond laser 3D direct writing (FsLDW) approach and the preparation of a protein micro-optic. (b) SEM images (on a

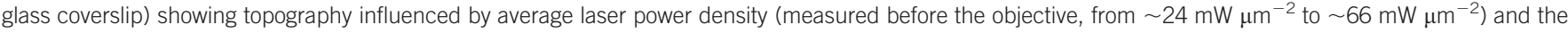
scanning step ( $100 \mathrm{~nm}, 150 \mathrm{~nm}$ and $200 \mathrm{~nm}$ ) when exposure time on single point is fixed at $1000 \mu \mathrm{s}$. The focal spot area where polymerization occurs is estimated to be $\sim 0.40 \mu \mathrm{m}^{2}$. Scale bar $=2 \mu \mathrm{m}$. (c) SEM images of protein micro-KPLs on a glass coverslip with thicknesses of $\sim 1 \mu \mathrm{m}$ but different diameters of (1) $50 \mu \mathrm{m}$, (2) $60 \mu \mathrm{m}$ and (3) $80 \mu \mathrm{m}$. (4) AFM characterization exhibiting 3D morphology and $\sim 10-\mathrm{nm}$ average roughness of the protein micro-KPL (with a diameter of $\sim 60 \mu \mathrm{m}$ ). The unit of the coordinates is $\mu \mathrm{m}$. (5) The cross section at the central line of the protein micro-KPL in (4) by AFM. $H$ stands for the thickness of the protein micro-KPL. Scale $\mathrm{bar}=10 \mu \mathrm{m}$. AFM, atomic force microscope; 3D, three-dimensional; FsLDW, femtosecond laser direct writing; micro-KPL, microscale kinoform phase-type lens; SEM, scanning electron microscopy. 
a

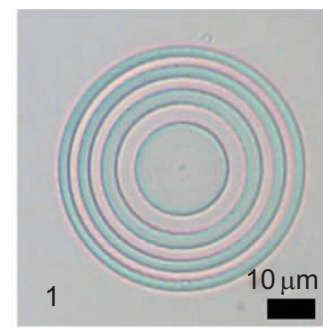

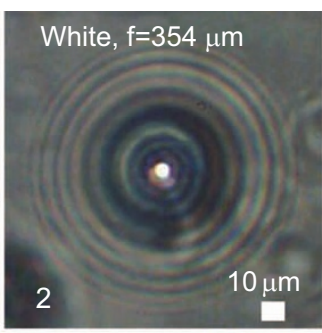

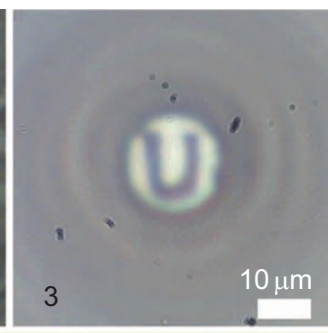

4

\section{$100 \mu \mathrm{m}$}

b

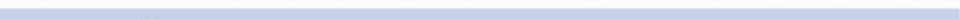

1

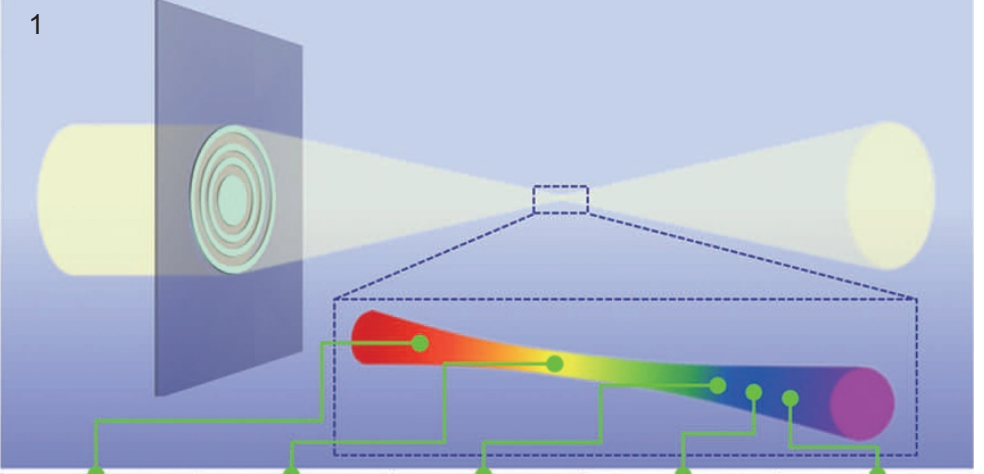

2
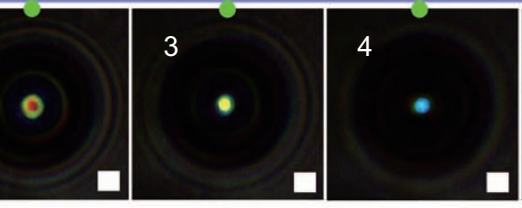

5

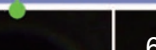

6

$$
\text { c }
$$

c 460
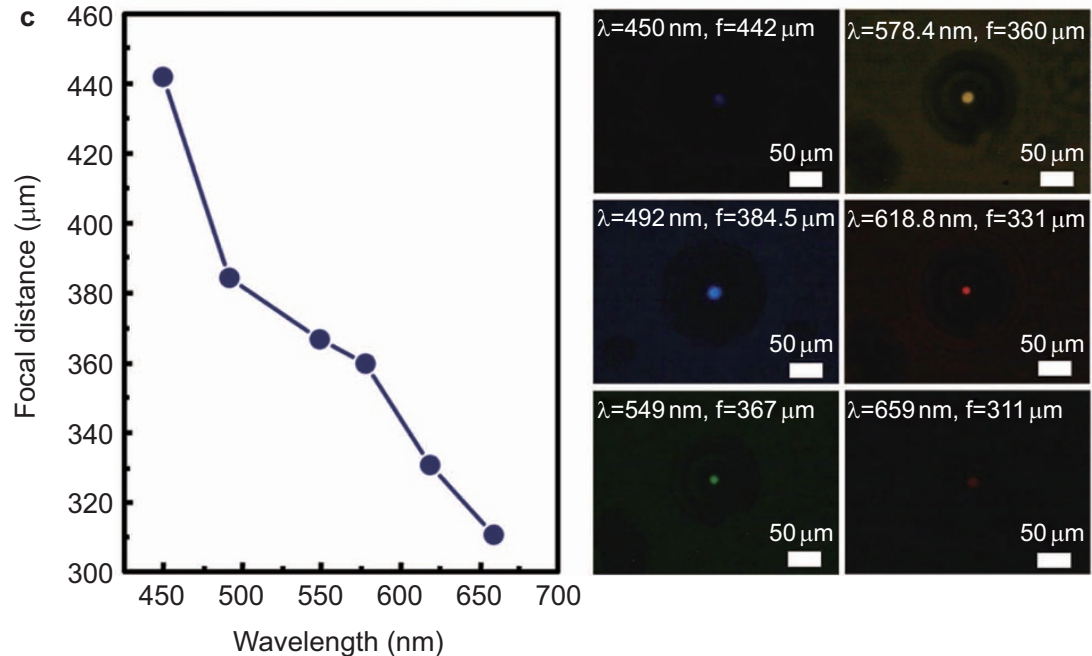

$50 \mu \mathrm{m}$
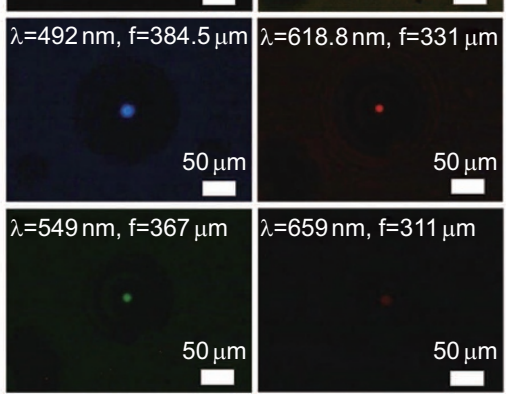

Figure 2 (a-1) The protein micro-KPL with a diameter of $50 \mu \mathrm{m}$ fabricated on a glass coverslip. (a-2) The focusing images of the protein micro-KPL in a-1 in air under illumination with white light from a halogen lamp. The focal length is measured to be $\sim 354 \mu \mathrm{m}$. (a-3) Optical microscope characterization of the imaging of the protein micro-KPL in a-1. (a-4) The focusing and shaping of a 532-nm-laser beam by the protein micro-KPL with a diameter of $100 \mu \mathrm{m}$ when immersed in Rhodamine B aqueous solution. (b-1) The minus chromatic dispersion of a protein micro-KPL in air. The schematic shows that the white light is dispersed along the optical axis. (b-2b-6) The optical microscope images of the focal spots of several monochromatic lights contained in the white incident light, caught at different locations on the optical axis under white light illumination. Scale bar $=20 \mu \mathrm{m}$. (c) The focal lengths under illumination of monochromatic lights with different wavelengths and the corresponding curve of the protein micro-KPL in a-1. micro-KPL, microscale kinoform phase-type lens. 
femtosecond laser beam (titanium-sapphire laser, Spectra Physics 3960$\mathrm{X} 1 \mathrm{BB}, 80 \mathrm{MHz}$ repetition rate, $120 \mathrm{fs}$ pulse width, $800 \mathrm{~nm}$ central wavelength) was tightly focused in the ink by a high-numerical aperture $(\mathrm{NA}=1.35)$ oil-immersion objective $(60 \times)$. Because the two-photoninduced polymerization of the BSA molecules was confined to the core region of the laser focal spot, high laser energy density over the threshold of the two-photon absorption can guarantee nanoscale precision for the BSA hydrogel microstructures (with $\leqslant 200 \mathrm{~nm}$ minimum line width here. ${ }^{27}$ ) To achieve three-dimensional (3D) microstructures, a piezo stage with 1-nm precision (PI P-622 ZCD) was used to control the sample's vertical movements. Simultaneously, the beam's horizontal scanning was performed by a two-galvano-mirror set. The complicated $3 \mathrm{D}$ geometry of the hydrogel microstructures was first designed in $3 \mathrm{ds}$ Max and then converted to computer processing data to control the 3D scanning. After the laser fabrication and water-rinsing steps, the asformed protein micro-KPLs can be obtained on the matrix (see Figure la and Scheme S1 in Supplementary Information).

Because the excellent surface quality and 3D geometry quality are the prerequisites for the optical properties of protein micro-KPLs, the processing parameters have to be carefully optimized. ${ }^{33}$ Here, a high concentration of BSA in the ink $\left(600 \mathrm{mg} \mathrm{mL}^{-1}\right)$ was utilized to obtain a high crosslinking density inside protein hydrogels, which helps achieve excellent optical and mechanical properties. In fact, numerous FsLDW parameters, including the scanning step, laser power density, and exposure time on a single point, play important roles in determining the quality of the 3D geometry and the quality of the surface of the protein hydrogel microstructures, as shown in Figure 1b, and, furthermore, the final properties of the protein micro-KPLs. Herein, the exposure time on a single point was fixed at $1000 \mu$ s. When the scanning step is $100 \mathrm{~nm}$ or $150 \mathrm{~nm}$, the laser power density should be about $48-50 \mathrm{~mW} \mathrm{~m}^{-2}$. In our work, to achieve rapidly direct writing, a scanning step of $200 \mathrm{~nm}$ and a laser power density of $\sim 60 \mathrm{~mW} \mu \mathrm{m}^{-2}$ were applied (Figure $1 \mathrm{~b}$ ). The optimal processing parameters were chosen based on the comprehensive quality of the protein microstructures shown in Figure $1 \mathrm{~b}$ (considering the quality of both the surface and 3D geometry). As a result, the protein micro-KPLs with diameters of $50 \mu \mathrm{m}$, $60 \mu \mathrm{m}$ and $80 \mu \mathrm{m}$ could be completed with high quality in several minutes (Figure 1c-1-1c-3). The surface roughness of the protein micro-KPLs in air was approximately $10 \mathrm{~nm}$, which was demonstrated by atomic force microscope (AFM), as shown in Figure 1c-4. Figure 1c-5 shows the cross section at the central line of the protein micro-KPL shown in Figure 1c-4 by AFM and shows that the 3D controllability of FsLDW is good. Such smooth surface morphology together with welltailored 3D geometry can ensure high optical performances.

Here, the optical performances of the BSA micro-KPLs were evaluated by an upright optical microscope (Motic BA400). The pictures of imaging, focusing and chromatic dispersion were taken by properly tuning the positions of the samples along the light axis. A halogen lamp provided white light illumination, and monochromatic incident lights with different wavelengths were obtained by filtering white light with narrow-band filters (20-nm bandwidth and central wavelengths of $450 \mathrm{~nm}, 578.4 \mathrm{~nm}, 492 \mathrm{~nm}, 618.8 \mathrm{~nm}, 549 \mathrm{~nm}$ and $659 \mathrm{~nm})$. The scanning electron microscopy (SEM) characterization was carried out using a field emission scanning electron microscope (JSM-7500F; JEOL, JEOL Ltd., Tokyo, Japan), and the samples were sputter-coated with Au film with a thickness of $\sim 10 \mathrm{~nm}$ (at a current of $20 \mathrm{~mA}$ for $60 \mathrm{~s}$ ) using an auto fine coater (JFC-1600; JEOL). The high BSA concentration (up to $600 \mathrm{mg} \mathrm{mL}^{-1}$ ) in the processing ink and the carefully optimized FsLDW parameters resulted in a high crosslinking degree and sufficient solidity of the protein microhydrogels. Therefore, a secondary crosslinking by chemical methods, which has often been used in previous reports of protein FsLDW, was not needed. ${ }^{34}$ Thus, protein microhydrogels were analyzed by SEM immediately after drying in the air to faithfully characterize the BSA microhydrogels in different experiment procedures (Figures 1 and 3 and Supplementary Fig. S1).

\section{RESULTS AND DISCUSSION}

\section{Optical characteristics of protein micro-KPLs}

The focusing and imaging performance of protein micro-KPLs were investigated by optical microscopy. As shown in Figure 2a, the distinct diffractive rings of micro-KPLs were apparent under illumination with white light from a halogen lamp. Using a protein micro-KPL with a size of $100 \mu \mathrm{m}$, a 532-nm-laser beam can be efficiently focused and shaped. To demonstrate the focusing and shaping performance of the laser, the protein micro-KPL was immersed in Rhodamine B aqueous solution; then, the laser beam was directed through the micro-KPL into the solution. By 532-nm excitation under an fluorescence microscope, the focused and shaped laser beam can easily be distinguished in the fluorescence image (Figure 2a-4). However, the incident light of the longer wavelength is more significantly bent, and the focal length is smaller, according to $f \sim 1 / \lambda$. As shown in Figure $2 b-1$, when a beam of white light was passing through a protein micro-KPL, the monochromatic light with a longer wavelength was focused more tightly with a smaller focal length, which is the so-called 'minus chromatic dispersion' behavior. More details are shown in Figure 2b-2 through Figure 2b-6, which present optical images of the focal spots of monochromatic lights with different wavelengths at different locations along the optical axis upon white-light illumination. By plotting the measured focal lengths with the wavelengths of monochromatic incident lights, the typical minus chromatic dispersion effect is shown in Figure $2 \mathrm{c}$. The focal length decreased from $\sim 442 \mu \mathrm{m}$ to $\sim 311 \mu \mathrm{m}$ by tuning the wavelength from $450 \mathrm{~nm}$ to $659 \mathrm{~nm}$.

\section{$\mathrm{pH}$-independent optical properties of protein micro-KPLs}

As reported earlier, ${ }^{26,27}$ protein micro-hydrogels are sensitive to the $\mathrm{pH}$ value of surroundings. When the $\mathrm{pH}$ value is higher or lower than its $\mathrm{pI}$ ( $\mathrm{pI}$ is the $\mathrm{pH}$ value under which the protein molecules are electrically neutral), the protein hydrogel will swell due to the repulsion interaction between the deprotonated carboxylate or amine group under different $\mathrm{pH}$ values. In our previous results, the $\mathrm{pH}$ effect played a significant role in the optical properties of the protein microoptics, i.e., the protein spherical microlenses and harmonic diffractive relief microlenses. The focal length of protein microlenses can be easily adjusted by changing the $\mathrm{pH}$ value. However, such a $\mathrm{pH}$ effect is not always desirable for the application of protein micro-optics. In some cases, $\mathrm{pH}$ independence is required. Therefore, how to suppress the effects of $\mathrm{pH}$-induced swelling or shrinking on optical properties of protein micro-optics is an open question.

It was found that the $\mathrm{pH}$-induced swelling and shrinking effects can be weakened by increasing the crosslinking degree of the protein hydrogel. ${ }^{34}$ In our work, using a high concentration of BSA solution (600 $\mathrm{mg} \mathrm{mL}^{-1}$ ) and optimizing the processing parameters, the crosslinking degree of the protein hydrogel could be increased. Consequently, the degree of swelling was reduced to some extent, which would be helpful for suppressing the $\mathrm{pH}$ response. More importantly, the structure of the micro-optics has to be designed to prevent the $\mathrm{pH}$ effect. For example, the focal length for protein micro-KPLs is determined by $f \approx r_{m}{ }^{2} / m \lambda$, which means that $r_{m}$ and $\lambda$ are two crucial factors in determining $f$. However, both parameters are $\mathrm{pH}$-independent. Although the swelling and shrinking effects may still occur in the 
a

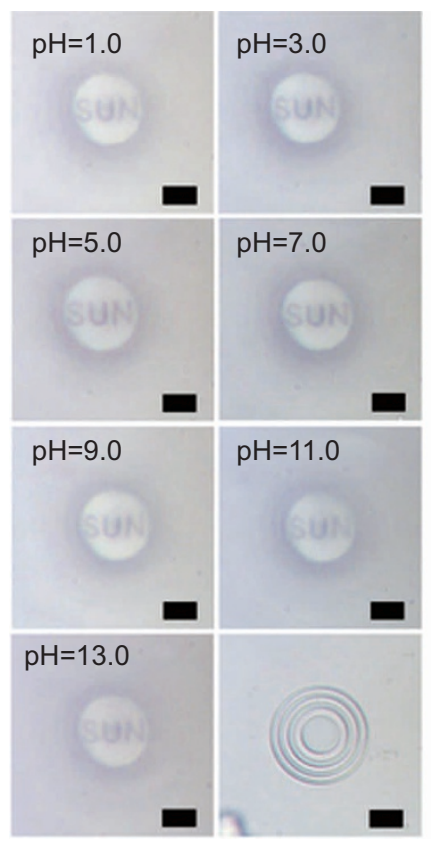

b
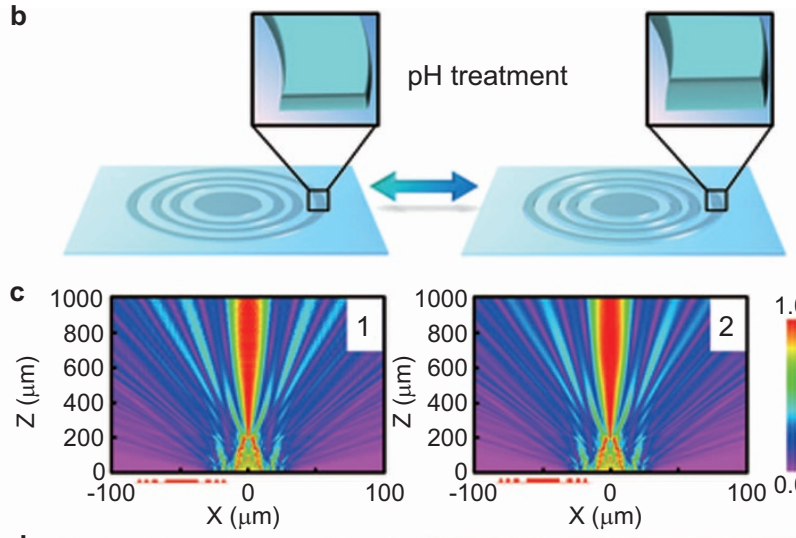

d

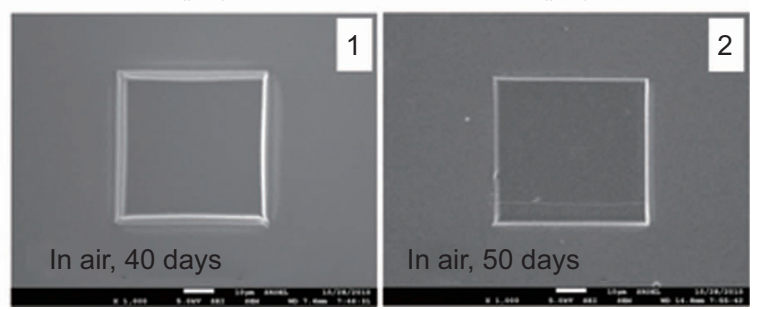

Figure 3 (a) Stable imaging and focusing feature of the protein micro-KPL on a glass coverslip in buffers with different values of $\mathrm{pH}$. The topography of the protein micro-KPL after pH treatment is the same as the original one. Scale bar $=20 \mu \mathrm{m}$. (b) The pH effect on morphology of a protein micro-KPL. The thickness, rather than the width, is changed owing to the restriction of the matrix. (c) Rsoft simulations of the focusing of a protein micro-KPL with a diameter of $60 \mu \mathrm{m}$ under neutral (1) and basic or acid buffers (2). The color scale bar represents the normalized light intensity (energy). (d) SEM images of BSA microsquares on a glass coverslip after long-term storage in air (40 days and 50 days). BSA, bovine serum albumin; micro-KPL, microscale kinoform phase-type lens; SEM, scanning electron microscopy.

protein micro-KPL, the $\mathrm{pH}$ effect can change the thickness of the protein zones but not $r_{m}$, which is mainly due to the restriction of the substrates. Therefore, the $r_{m}$ of a protein micro-KPL always remains constant during $\mathrm{pH}$ treatment (Figure $3 \mathrm{a}$ ). Thus, the unique structure of micro-KPLs can prominently block the $\mathrm{pH}$ effect. These assumptions are consistent with the experimental results. As shown in Figure $3 \mathrm{a}$, the protein micro-KPLs exhibit similar optical features and imaging results within a wide $\mathrm{pH}$ variation, from 1.0 to 13.0. During repeated $\mathrm{pH}$ treatment, the focused imaging did not change much. Such pH-independent behaviors can also be theoretically demonstrated by the Rsoft simulation. In Figure $3 c$, a protein micro-KPL with a diameter of $\sim 60 \mu \mathrm{m}$ was chosen for the simulation. The light energy dispersion of a protein micro-KPL to $550 \mathrm{~nm}$ light was calculated under different $\mathrm{pH}$ values, e.g., $\mathrm{pH}=7.0$ (Figure $3 \mathrm{c}-1$ ) and 13.0 or 1.0 (Figure $3 \mathrm{c}-2$ ). For both cases, the refractive index of the buffer $\left(n_{\text {buffer }}\right)$ was almost constant $(\sim 1.33)$, as measured by the Abbe refractometer. The refractive index of the BSA hydrogel $\left(n_{\text {hydrogel }}\right)$ was obtained from our previous work..$^{26,27}$ In the case of $\mathrm{pH}=7.0, h$ (the micro-KPL's thickness) $=1 \mu \mathrm{m}, n_{\text {hydrogel }}=1.5$ and $n_{\text {buffer }}=1.33$ (Figure $3 \mathrm{c}-1$ ). In addition, in the case of $\mathrm{pH}=13.0$ or $1.0, h=2 \mu \mathrm{m}$, $n_{\text {hydrogel }}=1.43$ and $n_{\text {buffer }}=1.33$ (Figure $3 c-2$ ). The simulation results are shown in Figure 3. The two light energy dispersion simulations, as shown in Figure $3 c-1$ and $3 c-2$, were almost the same for different $\mathrm{pHs}$ and a moderately changing thickness of the micro-KPLs, which indicates that the optical properties of the protein micro-KPLs are relatively stable against a strong acid or base and a large $\mathrm{pH}$ change.

\section{Long-term stability and biodegradability of protein micro-KPLs}

The long-term stability of protein microstructures in air was demonstrated by SEM. As shown in Figure 3d, after incubation in air for 40 days, 50 days or even longer, the smooth surface and the 3D geometry of the protein microstructures did not change at all, indicating that these micro-devices have long-term stability of original topography and performances in air. In addition, similar behaviors of the protein microstructures can be observed in pure water. Obviously, the longterm stability in common operation environments greatly assists the practical application of the protein-based micro-optics.

The biodegradability of the protein microhydrogels was also investigated by SEM. After immersing them in rainwater (which contains various bacteria) under room temperature $\left(\sim 22{ }^{\circ} \mathrm{C}\right)$ for a week, it could be observed that many bacteria grew and adhered to the surface of the protein microstructures, as shown in Figure $4 \mathrm{~b}$ and $4 \mathrm{e}$. The enlarged view in Figure 4e exhibited obvious degradation phenomena. The original smooth surface of the microstructures was obviously
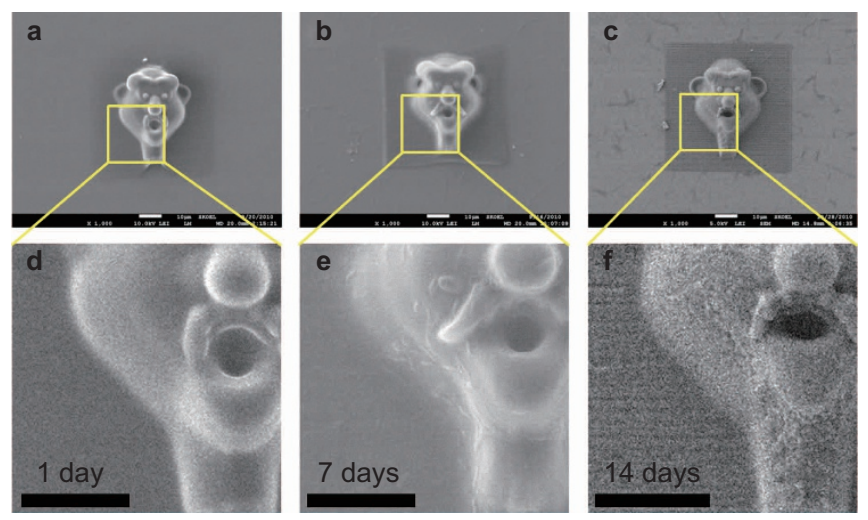

Figure 4 Biodegradation of BSA microhydrogels on a glass coverslip in rainwater at room temperature $\left(\sim 22{ }^{\circ} \mathrm{C}\right)$. (a-c) SEM images of the BSA microhydrogel in rainwater at $\sim 22^{\circ} \mathrm{C}$ for 1 day, 7 days and 14 days, respectively. (d-f) SEM images of partially enlarged details of $\mathbf{a}-\mathbf{c}$, respectively. Scale bar $=10 \mu \mathrm{m}$. BSA, bovine serum albumin; SEM, scanning electron microscopy. 
a

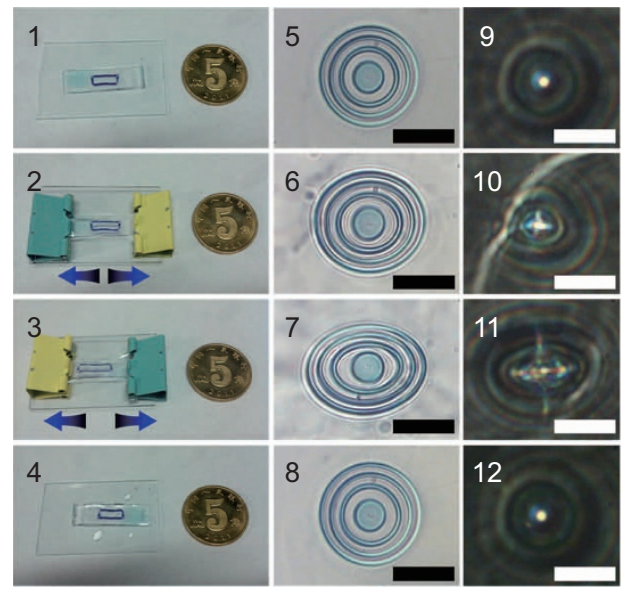

b
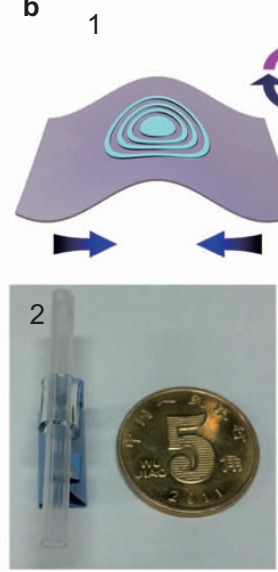
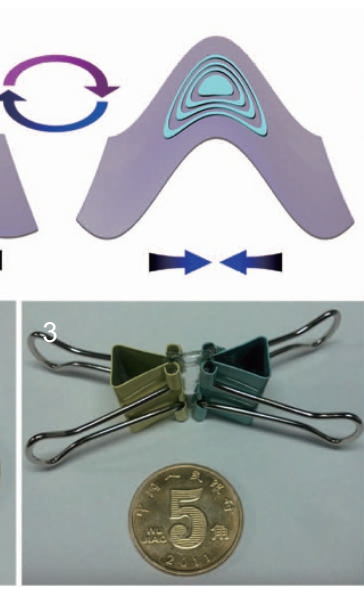

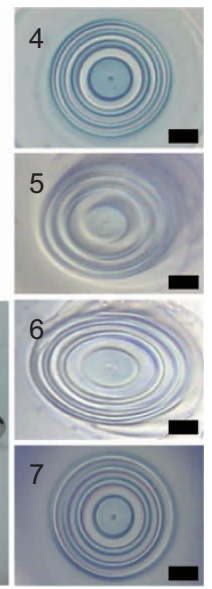

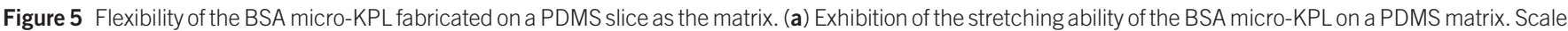

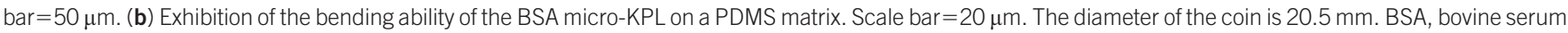
albumin; micro-KPL, microscale kinoform phase-type lens; PDMS, polydimethylsiloxane.

damaged and vanished after 2 weeks (Figure 4c and 4f), and the microstructures could totally biodegrade, collapse and even disappear after several months. The biodegradation happened in an aqueous surrounding with microorganisms that cause decomposition, which indicated that the biodegradable behavior could be controllably triggered by proper external environmental conditions. These results confirmed that the protein-based micro-hydrogels fabricated by FsLDW can also be utilized as biodegradable micro-devices.

\section{Flexibility and stretchability of protein micro-KPLs}

As previously shown, the protein microhydrogels have a soft nature because their Young modulus is as low as $\sim 4 \mathrm{MPa}{ }^{33}$ The protein microhydrogels are so soft that they can be used for flexible and stretchable optics/photonics if incorporated with suitable soft matrixes. For this reason, flexible PDMS slices were used as the matrixes where the protein micro-KPLs can be constructed by FsLDW. Then, the as-formed protein micro-KPLs on PDMS substrates exhibited interesting flexibility. First, the stretchability of the protein micro-KPLs was demonstrated by applying an external drawing force. The exhibition of the original shape of the sample, the microscopy image and the focusing test of a protein micro-KPL with a diameter of $80 \mu \mathrm{m}$ are shown in Figure $5 \mathrm{a}-1,5 \mathrm{a}-5$ and $5 \mathrm{a}-9$, respectively. By stretching, the length of the sample was increased to $\sim 90 \mu \mathrm{m}$, which was approximately 1.1 times longer than the original (Figure 5a2 and 5a-6). Then, the sample could be stretched to $\sim 101 \mu \mathrm{m}$, or even larger (Figure 5a-3 and 5a-7). During the stretching process, the focal spot of the sample was deformed along with the transformation, as demonstrated in Figure 5a-9-5a-11. Once releasing the applied stress, the sample would immediately recover to the original state (Figure 5a4 ), and the focusing performance was not damaged at all (Figure 5a-12). However, the bending flexibility of the sample was tested according to the method shown in Figure 5b-1. The sample was first bent and then adhered to a circular tube with a diameter of $\sim 5 \mathrm{~mm}$ (Figure 5b-2). Due to the support of the curved surface, the size of the sample was elongated to $\sim 1.2$ times longer than the original length (Figure $5 b-5$ ). Furthermore, the PDMS slice with protein micro-KPLs was directly folded (Figure 5b-3), and the micro-KPL's size was increased to $\sim 1.4$ times longer than the original length (Figure 5b-6). Clear focusing images of the bent micro-KPL were difficult to obtain because different parts were not on the same plane and some were beyond the focus due to bending (Figure 5b-5 and 5b-6). Once the external bending force was removed, the initial state could rapidly be recovered, without any changes in topography or optical performances (Figure 5b-7).

\section{CONCLUSIONS}

In summary, a novel soft and biodegradable protein micro-KPL has been fabricated on PDMS matrixes by a home-made FsLDW system. Three key points are achieved in this work simultaneously. First, excellent processing quality and optical performances for practical applications are achieved. Due to the excellent surface quality and 3D geometry, the protein micro-KPLs exhibited ideal and distinctive optical properties, including the minus chromatic aberration profile, higher efficiency than the Fresnel zone plate and good imaging and focusing features. Second, the soft property of protein microhydrogels was utilized to obtain soft protein microelements for increasingly important flexible microsystems. As-formed protein micro-KPLs have excellent flexibility and stretchability, and their optical properties were not damaged after repeated stretching or bending (up to $\sim 40 \%$ length change, or even greater by stretching or bending). Third, the valuable balance between the biodegradation in natural aqueous environments and sufficient stability in common operation environments, required for the utilization of protein micro-KPL as an environment-friendly and practical micro-optical element, was maintained. For example, in rainwater with a variety of microorganisms, preliminary but obvious biodegradation occurred after $\sim 14$ days, but in pure water and air, the sample was unchanged after more than 50 days. In contrast to the previously reported protein microlenses, the optical properties of the protein micro-KPLs were hardly influenced by $\mathrm{pH}$ value (from 1.0 to 13.0) due to their optical features, which ensure that they would work properly in relatively harsh conditions (e.g., in strong acid or base) and in occasions with no need for stimuli-response. The organic combination of the three points by the FsLDW approach led to the realization of protein-based 'green' soft micro-optics with diverse merits such as being biocompatible and environmentally friendly. Such soft protein micro-optics would have great potential in applications such as flexible and stretchable photonics and optics as well as soft integrated optical microsystems.

\section{ACKNOWLEDGEMENTS}

HBS thanks the National Science Foundation of China (Grant No. 90923037) and the National Basic Research Program of China (973 Program) (Grant No. 2011CB013005) for support. WFD thanks the National Science Foundation of 
China (Grant Nos. 91123029, 61077066, 61137001 and 61127010) and the 863 Project of China (Grant No. 2012AA063302) for financial support.

1 Dong L, Agarwal AK, Beebe DJ, Jiang HR. Adaptive liquid microlenses activated by stimuli-responsive hydrogels. Nature 2006; 442: 551-554.

2 Rogers JA, Bao ZN, Baldwin K, Dodabalapur A, Crone B et al. Paper-like electronic displays: large-area rubber-stamped plastic sheets of electronics and microencapsulated electrophoretic inks. Proc Natl Acad Sci USA 2001; 98: 4835-4840.

3 Sirringhaus $\mathrm{H}$, Tessler N, Friend $\mathrm{RH}$. Integrated optoelectronic devices based on conjugated polymers. Science 1998; 280: 1741-1744.

4 Kim JY, Lee K, Coates NE, Moses D, Nguyen TQ et al. Efficient tandem polymer solar cells fabricated by all-solution processing. Science 2007; 317: 222-225.

5 Shrotriya V. Polymer power. Nat Photonics 2009; 3: 447-449.

6 Taylor RA, Otanicar T, Rosengarten G. Nanofluid-based optical filter optimization for PV/T systems. Light Sci App/2012; 1: e34; doi:10.1038/lsa.2012.34.

7 Monat C, Domachuk P, Eggleton BJ. Integrated optofluidics: a new river of light. Nat Photonics 2007; 1: 106-114.

8 Dai D, Bauters J, Bowers JE. Passive technologies for future large-scale photonic integrated circuits on silicon: polarization handling, light non-reciprocity and loss reduction. Light Sci App/2012; 1: e1; doi:10.1038/lsa.2012.1.

9 Hwang SW, Tao H, Kim DH, Cheng HY, Song JK et al. A physically transient form of silicon electronics. Science 2012; 337: 1640-1644.

10 Mannoor MS, Tao H, Clayton JD, Sengupta A, Kaplan DL et al. Graphene-based wireless bacteria detection on tooth enamel. Nat Commun 2012; 3: 763.

$11 \mathrm{Kim}$ RH, Tao H, Kim TI, Zhang YH, Kim S et al. Materials and designs for wirelessly powered implantable light-emitting systems. Small 2012; 8: 2812-2818.

12 Kim S, Mitropoulos AN, Spitzberg JD, Tao H, Kaplan DL et al. Silk inverse opals. Nat Photonics 2012; 6: 818-823.

13 Parker ST, Domachuk P, Amsden J, Bressner J, Lewis JA et al. Biocompatible silk printed optical waveguides. Adv Mater 2009; 21: 2411-2415.

14 Amsden JJ, Domachuk P, Gopinath A, White RD, Negro LD et al. Rapid nanoimprinting of silk fibroin films for biophotonic applications. Adv Mater 2010; 22: 1746-1749.

15 Lawrence BD, Cronin-Golomb M, Georgakoudi I, Kaplan DL, Omenetto FG. Bioactive silk protein biomaterial systems for optical devices. Biomacromolecules 2008; 9 : 1214-1220.

16 Lin DM, Tao H, Trevino J, Mondia JP, Kaplan DL et al. Direct transfer of subwavelength plasmonic nanostructures on bioactive silk films. Adv Mater 2012; 24: 6088-6093.

17 Kim RM, Lu NS, Ma R, Kim YS, Kim RH et al. Epidermal electronics. Science 2011; 333: 838-843.

18 John G, Jadhav SR, Menon VM, John VT. Flexible optics: recent developments in molecular gels. Angew Chem Int Ed 2012; 51: 1760-1762.
19 Vidyasagar A, Handore K, Sureshan KM. Soft optical devices from self-healing gels formed by oil and sugar-based organogelators. Angew Chem Int Ed 2011; 50: 80218024.

20 Ghosh S, Reddy CM. Elastic and bendable caffeine cocrystals: implications for the design of flexible organic materials. Angew Chem Int Ed 2012; 51: 10319-10323.

21 LaFratta CN, Fourkas JT, Baldacchini T, Farrer RA. Multiphoton fabrication. Angew Chem Int Ed 2007; 46: 6238-6258.

22 Kawata S, Sun HB, Tanaka T, Takada K. Finer features for functional microdevice. Nature 2001; 412: 697-698.

23 Xiong W, Zhou YS, He XN, Gao Y, Mahjouri-Samanil M et al. Simultaneous additive and subtractive three-dimensional nanofabrication using integrated two-photon polymerization and multiphoton ablation. Light Sci Appl 2012; 1: e6; doi:10.1038/Isa.2012.6.

24 Zhang YL, Chen QD, Xia H, Sun HB. Designable 3D nanofabrication by femtosecond laser direct writing. Nano Today 2010; 5: 435-448.

25 Basu S, Wolgemuth CW, Campagnola PJ. Enzymatic activity of alkaline phosphatase inside protein and polymer structures fabricated via multiphoton excitation. Biomacromolecules 2004; 5: 2347-2357.

26 Sun YL, Dong WF, Yang RZ, Meng X, Zhang L et al. Dynamically tunable protein microlenses. Angew Chem Int Ed 2012; 124: 1590-1594.

27 Sun YL, Liu DX, Dong WF, Chen QD, Sun HB. Tunable protein harmonic diffractive micro-optical elements. Opt Lett 2012; 37: 2973-2975.

28 Kaehr B, Allen R, Javier DJ, Currie J, Shear JB. Guiding neuronal development with in situ microfabrication. Proc Natl Acad Sci USA 2004; 101: 16104-16108.

29 Cho KC, Lien CH, Lin CY, Chang CY, Huang LL et al. Enhanced two-photon excited fluorescence in three-dimensionally crosslinked bovine serum albumin microstructures. Opt Express 2011; 19: 11732-11739.

30 Lin $\mathrm{CY}$, Lien $\mathrm{CH}$, Cho KC, Chang $\mathrm{CY}$, Chang NS et al. Investigation of two-photon excited fluorescence increment via crosslinked bovine serum albumin. Opt Express 2012; 20: 13669-13676.

31 Pitts JD, Campagnola PJ, Epling GA, Goodman SL. Submicron multiphoton free-form fabrication of proteins and polymers: studies of reaction efficiencies and applications in sustained release. Macromolecules 2000; 33: 1514-1523.

32 Chen QD, Wu D, Niu LG, Wang J, Lin XF et al. Phase lenses and mirrors created by laser micronanofabrication via two-photon photopolymerization. Appl Phys Lett 2007; 91 : 171105.

33 Khripin CY, Brinker CJ, Kaehr B. Mechanically tunable multiphoton fabricated protein hydrogels investigated using atomic force microscopy. Soft Matter 2010; 6: 2842 2848

34 Kaehr B, Shear JB. Multiphoton fabrication of chemically responsive protein hydrogels for microactuation. Proc Natl Acad Sci USA. 2008; 105: 8850-8854.

\section{(c) (1) (2) This work is licensed under a Creative Commons Attribution- (c) $\mathrm{BY}$ NG $\mathrm{SA}$ NonCommercial-ShareAlike 3.0 Unported license. To view a copy of this license, visit http://creativecommons.org/licenses/by-nc-sa/3.0}

